\title{
Efficient and Safe Application of a FOLFIRI/Bevacizumab Combination to a Patient with Locally Advanced Rectal Cancer and Severe Chronic Renal Failure
}

\author{
Irfan Cicin Hakan Karagol Sernaz Uzunoglu Kazım Uygun \\ Department of Medical Oncology, Faculty of Medicine, Trakya University, Edirne, Turkey
}

Cytotoxic treatment of patients with advanced or metastatic cancer, undergoing hemodialysis due to chronic renal failure, still remains a problem due to a lack of sufficient pharmacokinetic and pharmacodynamic data on most cytotoxic agents [1]. A number of articles have been published on the safe use of various agents, such as 5-flourouracil (5-FU), folinic acid (FA), irinotecan (CPT-11) and oxaliplatin, in patients suffering from advanced or metastatic cancer and undergoing hemodialysis due to chronic renal failure [2-5]. However, it is still unclear whether bevacizumab (BEV) - a new agent with an IgG1 structure - can be safely used. After a review of the literature, we have observed that FOLFOX4, FOLFIRI regimens and BEV can be safely and efficiently used in these patients [1-6]. We report the case of a 38 -year-old male who suffered from severe chronic renal failure and local irresectable recurrent rectal cancer. The patient was admitted to our hospital with stage II cecal cancer in January 1999. After a hemicolectomy, the patient did not receive any adjuvant therapy. Then, in August 2003, he was diagnosed with stage III rectal cancer (metachronous cancer). After surgical removal of the tumor, postoperative radiotherapy and chemotherapy of intravenous bolus 5-FU/FA was applied (Mayo protocol). Later, in December 2005, a locally recurrent irresectable rectal cancerous lesion and subsequent postrenal azotemia emerged. Due to the postrenal azotemia and bilateral hydroureteronephrosis, bilateral percutaneous nephrostomy were performed. After that, the glomerular filtration rate (GFR) was determined as $28 \mathrm{ml} / \mathrm{min}$. The patient received 2 cycles of standard FOLFOX4 regimen. Due to a grade 3-4 hematological toxicity, the therapy was discontinued after the second cycle. Due to a blockage in both nephrostomies, both catheters had to be replaced. The patient received hemodialysis over a period of 1 month. He was put on a biweekly schedule of dose-reduced CPT-11 (100 mg/m², $180 \mathrm{mg}$ in total) on day 1 , standard dose 5-FU $\left(400 \mathrm{mg} / \mathrm{m}^{2}, 720 \mathrm{mg}\right.$ in total as i.v.bolus and $600 \mathrm{mg} / \mathrm{m}^{2}$,
$1,080 \mathrm{mg}$ in total, as i.v. infusion for $22 \mathrm{~h}$ on days 1 and 2), standard dose FA $\left(200 \mathrm{mg} / \mathrm{m}^{2}, 360 \mathrm{mg}\right.$ in total, as i.v. infusion for $2 \mathrm{~h}$ on days 1 and 2) and BEV $5 \mathrm{mg} / \mathrm{kg}$ (total $300 \mathrm{mg}$ ) on day 1 (FOLFIRI/BEV regimen). With the nephrostomies functioning efficiently, the patient did not require hemodialysis after the first cycle of FOLFIRI/BEV (GFR $25 \mathrm{ml} / \mathrm{min}$ ). Due to excellent tolerability and a lack of severe side effects, the dose of CPT-11 was increased to biweekly $125 \mathrm{mg} / \mathrm{m}^{2}$ (225 $\mathrm{mg}$ in total), $150 \mathrm{mg} / \mathrm{m}^{2}$ (270 $\mathrm{mg}$ in total), and $180 \mathrm{mg} / \mathrm{m}^{2}$ (320 mg in total). Hematological and non-hematological (such as proteinuria and hypertension) toxicities grade 3/4 were not observed. A total of 8 cycles FOLFIRI/BEV were applied over 4 months of treatment. The patient achieved regression (partial response) and clinical improvement.

This is the first case report to demonstrate the feasibility and efficacy of a biweekly treatment with a full-dose FOLFIRI/ $\mathrm{BEV}$ regimen in a patient with metastatic rectal cancer and severe chronic renal failure.

\section{References}

1 Eneman JD, Philips GK: Cancer management in patients with end-stage renal disease. Oncology (Williston Park) 2005;19:1199-1212.

2 Shinozaki E, Mizunuma N, Tanabe M, Chin K, Ota K, Ohkochi N, Hatake K: Induction of CPT-11 in a patient on hemodialysis with metastatic rectal cancer. Gan To Kagaku Ryoho 2005;32:397-399.

3 Stemmler J, Weise A, Hacker U, Heinemann V, Schalhorn A: Weekly irinotecan in a patient with metastatic colorectal cancer on hemodialysis due to chronic renal failure. Onkologie 2002;25:60-63.

4 Honecker FU, Brummendorf TH, Klein O, Bokemeyer C: Safe use of oxaliplatin in a patient with metastatic breast cancer and combined renal and hepatic failure. Onkologie 2006;29:273-275.

5 Mir O, Alexandre J, Ropert S, Amsellem-Ouazana D, Flam T, Beuzeboc P, Zerbib M, Debre B, Guillevin L, Goldwasser F: Combination of gemcitabine and oxaliplatin in urothelial cancer patients with severe renal or cardiac comorbidities. Anticancer Drugs 2005;16:1017-1021.

6 Abdelkefi A, Mellouli F, Bejaoui M: Treatment of a patient with chronic renal failure with rituximab for a follicular lymphoma: safe and successful option of rituximab therapy. Eur J Haematol 2003;71:128-129.

\begin{tabular}{ll}
\hline KARGER & @ 2007 S. Karger GmbH, Freiburg \\
Fax +497614 520714 & Accessible online at: \\
$\begin{array}{l}\text { E-mail Information@Karger.de } \\
\text { www.karger.com }\end{array}$ & www.karger.com/onk
\end{tabular}

